\title{
AUTOANTIBODIES ARE ASSOCIATED WITH DIFFERENT PATTERNS OF BRAIN VOLUME REDUCTION CHILDHOOD-ONSET SYSTEMIC LUPUS ERYTHEMATOSUS
}

Analice Alves Santana ${ }^{1}$, Willian Mikos dos Santos ${ }^{1}$, Renan Bazuco Frittoli ${ }^{1}$, Paulo Rogério Julio ${ }^{1}$, Roberto Marini ${ }^{1}$, Leticia Rittner ${ }^{1}$, Simone Appenzeller ${ }^{1, *}$

1.Universidade Estadual de Campinas, Campinas (SP), Brazil.

*Corresponding author: appenzellersimone@gmail.com

\section{BACKGROUND}

Brain volumes in childhood-onset systemic lupus erythematosus (CSLE) have been shown to be reduced when compared to healthy controls. While clinical features associated with atrophy and associations with medication have been described, the role of autoantibodies in volume reduction is still controversial.

\section{METHODS}

We included 76 consecutive cSLE patients [median age 18 years (range 10-27)] from a pediatric rheumatology outpatient unit and 77 healthy controls [median age 20.1 years (12-30), with similar sex and sociodemographic distribution]. All individuals underwent three-tesla magnetic resonance imaging (3T-MRI) exams. A complete clinical, laboratory and neurological evaluation was performed in all subjects. Neurological manifestations were analyzed according to the ACR classification criteria. Autoantibodies SLE patients were further assessed for clinical and laboratory SLE manifestations, disease activity [SLE disease activity index (SLEDAI)], damage [Systemic Lupus International Collaborating Clinics/American College of Rheumatology damage index (SDI)] and current drug exposures. Data were compared by nonparametric tests. Multivariate regression was done to identify independent variables associated with volume reduction.

\section{RESULTS}

Anti-P antibodies were observed in $10(13.1 \%)$ and associated with right and left amygdala volumes ( $p=0.02)$. Lupus anticoagulant was observed in $34(44.7 \%)$ and associated with bilateral amygdala $(p=0.02)$, hippocampal $(p=0.01)$ and cerebellar volume $(p=0.02)$. Anticardiolipin antibodies were observed in $22(28.9 \%)$ and associated with corpus callosum volume $(p=0.02)$. anti-Ro antibodies were identified in $22(28.9 \%)$ associated with cerebellar volume $(p=0.01)$.

\section{CONCLUSION}

Heterogeneous volume reduction is observed in CSLE patients. Positivity of autoantibodies and their specificity could be responsible for these findings and diverse clinical presentation. 